Marcella Costa

\title{
Fabbisogni e scelte linguistiche nella comunicazione turistica in Italia, con particolare riferimento alla lingua tedesca
}

\begin{abstract}
The paper reflects on the linguistic needs in the field of tourism communication in Italy starting from data on the main tourist markets of origin in Italy. After an overview on language choice in the pre-trip stage on national and regional level, it outlines a research project on language contact between German speaking tourists and hosts in Piedmont aiming at enhancing the quality of communication with German as a foreign language for tourism.
\end{abstract}

Keywords: linguistic needs, language choice in tourism, German as a foreign language for tourism, multilingual communication for tourism

\section{Le lingue straniere per la comunicazione turistica: considerazioni introduttive}

Secondo i dati dell'UNWTO ${ }^{1}$ il turismo è un settore economico che mostra, in Europa e nel mondo, un trend in continua ascesa. Per la destinazione Italia il Centro Internazionale di Studi sull'Economia Turistica prevede per il 2018 un aumento del 4,7\% degli arrivi mondiali, in particolare dall'Europa Centrale e dall'area extraeuropea ${ }^{2}$. Il risvolto linguistico della mobilità per scopi turistici è stato studiato nel corso degli ultimi decenni da molteplici prospettive, ponendo l'accento ora sulle caratteristiche della microlingua del turismo (ad es. per l'inglese: Nigro 2005, Gotti 2007, per lo spagnolo: Calvi 2006) ora su aspetti legati all'uso del web per la promozione turistica (Calvi 2004, Maci 2007), su specifici generi comunicativi (per il tedesco: Costa/Müller-Jacquier 2010, Fandrych/Thurmair 2010, Costa/Ravetto 2018), sulla descrizione della comunicazione turistica in termini di adattamento e accomodamento (Held 2018), sulla rilevanza della scelta della lingua di comunicazione (inglese vs. lingue locali e lingue dei turisti, cfr. Dannerer/Franz/Ortner 2017, Wilson

1 UNWTO World Tourism Barometer and Statistical Annex, June 2018.

http://mkt.unwto.org/ba rometer/june-2018-volume-16 [22.07.2018].

2 http://www.turismo.beniculturali.it/media/dati-turismo-2018/ [22.07.2018].

Marcella Costa, Università di Torino, Italia, marcella.costa@unito.it 
2018), sulle strategie di accomodamento (Goethals 2014) e di "commodificazione" delle lingue in contesti multilingui (Heller 2010, Jaworski/Thurlow 2010) o ancora sul valore di mercato del multilinguismo per il turismo (Duchêne/Piller 2011). Per quanto riguarda il tedesco, Reuter (2011) è stato il primo a puntare lo sguardo sulle potenzialità didattiche della lingua del turismo in direzione professionalizzante e in vista del rinnovamento dei curricula universitari di lingue e letterature straniere. Ancora esigui sono gli studi sull'impatto delle scelte linguistiche locali sull'efficacia della comunicazione turistica e sulla diversità linguistica in ambito turistico (cfr. Dannerer/Franz in questo volume).

In linea generale, la diversità linguistica può essere considerata sia come un ostacolo sia come un'opportunità: offrire contenuti turistici e interazioni in loco in più lingue oltre all'inglese richiede certamente forti investimenti in termini di localizzazione dei testi oltre che di formazione e reclutamento del personale, ma può avere ricadute importanti sul potenziale bacino di utenza e sul tessuto occupazionale di un aquesto volume e le riflessioni sul ruolo degli addetti multilingui del settore turistico in Duchêne 2009). Nello spazio linguistico europeo la comunicazione multilingue è un dato di fatto che dovrebbe trovare adeguato riscontro anche nelle pratiche comunicative in ambito turistico. Una riflessione sull'importanza della redazione dei contenuti in più lingue emerge in filigrana dal documento sulla digitalizzazione dell'offerta turistica pubblicato dal Ministero dei Beni e delle Attività Culturali e del Turismo $(\mathrm{MiBACT})^{3}$, che sottolinea anche l'importanza dell'adeguatezza sociolinguistica nella fase di localizzazione dei contenuti informativi.

In questo contributo, dopo una breve riflessione su fabbisogni e competenze per la comunicazione turistica multilingue (par. 2) e una disamina del grado di multilinguismo dei portali turistici italiani (par. 3), presenterò un progetto di ricerca e didattica che intende indagare il grado di multilinguismo nella comunicazione turistica in Piemonte e proporre interventi per migliorare le pratiche comunicative di accoglienza linguistica, con particolare riferimento alla lingua tedesca (par. 4).

\section{Fabbisogni linguistici e competenze per la comunicazione turistica multilingue}

Allo stato attuale non esistono ricerche sul fabbisogno linguistico nel comparto del turismo in Italia. Riportiamo qui di seguito i risultati di una ricerca esplorativa condotta nell'ambito del progetto SLEST (Standard Linguistico Europeo per il Settore Tu-

3 http://www.beniculturali.it/mibac/multimedia/MiBAC/documents/1460024515998_TD_Lab.pdf [22.07.2018]. 
ristico) che, attraverso interviste agli addetti del settore, ha messo in luce l'importanza della conoscenza di due lingue straniere e evidenziato la necessità di competenze comunicative differenziate in base ai profili professionali (competenza orale nel settore dell'accoglienza, competenza scritta e traduttiva nelle attività di agenzia). Gli addetti del settore intervistati nell'ambito del progetto SLEST dichiarano di conoscere una seconda lingua oltre l'inglese, sebbene quest'ultimo sia l'unico idioma che usano con sicura padronanza. La scarsa padronanza della seconda lingua straniera è in linea con i dati ISTAT ${ }^{4}$ sulla conoscenza delle lingue straniere dei cittadini italiani, che rivelano come l'obiettivo fissato dalle politiche linguistiche europee in materia di multilinguismo non sia ancora stato raggiunto ${ }^{5}$. Se si confrontano poi i dati ISTAT sulla seconda lingua appresa in Italia si nota un forte scollamento fra seconda lingua straniera studiata (per la fascia d'età 25-34 anni: francese 35\%, spagnolo 18\%, tedesco 9\%) e lingua dei principali paesi di provenienza dei flussi turistici: secondo l'Ente Italiano per il Turismo il primo paese di provenienza dei turisti stranieri in Italia è la Germania ${ }^{6}$, un dato che dovrebbe far riflettere sulla scelta della lingua da apprendere per chi viene formato in questo settore. Una cursoria ricognizione sulle lingue richieste da una delle principali agenzie interinali ${ }^{7}$ nel settore turistico-alberghiero sul territorio italiano evidenzia che i datori di lavoro in ambito turistico ricercano personale con competenza linguistica in almeno due lingue straniere (inglese e francese o tedesco). Accanto a questa combinazione esistono poi realtà locali in cui sono richieste altre lingue straniere (cfr. ad es. l'importanza crescente del russo come lingua del turismo).

Per descrivere gli effettivi fabbisogni linguistici è opportuno osservare sia il punto di vista del turista e delle sue aspettative in termini di "accoglienza linguistica" (Ammon 2015) e accomodamento (cfr. i contributi in Held 2018), sia il punto di vista delle destinazioni turistiche e la risposta che i diversi territori offrono in termini di scelte linguistiche, in riferimento alla redazione di testi in lingua straniera formulati per soddisfare le esigenze dei turisti nelle diverse fasi dell'esperienza di viaggio (fase di ispirazione, fase di pianificazione, fase di fruizione). A queste fasi corrispondono a livello di realizzazione linguistica una molteplicità di generi testuali, riassunti per la prima volta da Dann (1996) nelle macrocategorie di testi pre-trip e on-trip; i generi post-trip (recensioni, travelogue) assumono oggi un'importanza sempre maggiore, anche alla luce del loro impatto sulla reputazione delle destinazioni e delle strutture ricettive nell'ambito dell'economia collaborativa. L'insieme dei generi comunicativi

\footnotetext{
4 https://www.istat.it/it/files//2017/12/Report_Uso-italiano_dialetti_altrelingue_2015.pdf [22.07. 2018].

5 Gazzetta ufficiale della Comunità europea del 16/12/2008, Risoluzione del Consiglio del 21 novembre 2008, relativa a una strategia europea per il multilinguismo.

https://eur-lex.europa.eu/legal-content/IT/TXT/?uri=celex:32008G1216(01) [22.07.2018].

6 http://www.enit.it/it/studi.html [22.07.2018].

7 ADC ITCAT2800\%7c [15.05.2018].
} 
di ambito turistico richiede uno spettro ampio e diversificato di competenze nella lingua straniera per scopi professionali, quali:

- competenza traduttiva per la localizzazione dei siti, delle guide turistiche tradizionali, delle audioguide, delle informazioni rivolte ai turisti in loco (cartellonistica, menù, didascalie nei musei e siti di interesse);

- competenza di scrittura su supporto tradizionale e digitale per la gestione dei rapporti con i clienti;

- competenza di scrittura sui social media, ad es. per la gestione delle recensioni degli ospiti e per il management della reputazione;

- competenze orali per la gestione dell'interazione con i turisti in loco (attività di accoglienza alberghiera, attività informativa nei punti di accoglienza dedicati, visite guidate ecc.);

- competenza metacomunicativa per la riflessione sull'agire linguistico in contesto interculturale da parte degli operatori del settore.

Questo insieme di competenze può fornire la base per la progettazione di un curriculum universitario per la formazione linguistica di esperti della comunicazione turistica, da sviluppare nell'ambito dei corsi di laurea triennale e magistrale in Lingue e Culture per il Turismo per tutte le lingue oggetto di studio. Sulla necessità di proporre un curriculum linguistico consistente e orientato ai bisogni del settore si leggano le considerazioni critiche presenti nei resoconti del progetto SLEST, basate su una comparazione a livello europeo della formazione linguistica universitaria in ambito turistico, da cui emerge che la Spagna è l'unico paese europeo a offrire una formazione linguistica coerentemente strutturata per le professioni del turismo.

\section{Scelte linguistiche nella fase pre-trip}

Secondo un'indagine della European Tourism Association citata da Moraldo (in questo volume), nella fase di scelta e acquisto del prodotto turistico i consumatori prediligono contenuti redatti nella lingua materna, un comportamento che rende sempre più fondamentale la localizzazione dei siti web. Tale preferenza è confermata anche per la fase on-trip da Goethals (2014), che analizza il giudizio dei turisti spagnoli sulla competenza linguistica degli addetti del settore e sulla capacità di accomodamento linguistico in diverse destinazioni e, per i turisti tedeschi, dalla scheda paese ENIT-Germania (2017), che cita la conoscenza delle lingue fra i fattori determinanti nella scelta della destinazione. Sebbene sia probabile che nella fase on-trip i turisti tedescofoni siano maggiormente disponibili a utilizzare l'inglese come lingua franca rispetto, ad es., a quelli spagnoli (cfr. Goethals 2016), nella fase di pianificazione la presenza di materiale informativo nella lingua materna gioca un ruolo fon- 
damentale, come confermato da riscontri verbali avuti da turisti tedescofoni interessati al turismo montano, i quali riferiscono di avere infine optato per destinazioni, anche di nicchia, promosse attraverso il canale digitale in lingua tedesca.

Una prima riflessione sul grado di multilinguismo nella comunicazione pre-trip può essere fatta osservando le scelte di localizzazione dei portali informativi di alcune Agenzie turistiche locali (ATL), selezionate in base alla distribuzione geografica e agli interessi della ricerca specifica che verrà presentata nel par. 4. Oltre che in italiano i portali web delle ATL offrono i contenuti nelle seguenti lingue:

Tab. 1: Scelte linguistiche dei portali informativi di alcune destinazioni turistiche italiane.

\begin{tabular}{|c|c|c|c|c|c|c|c|c|c|}
\hline & $\begin{array}{l}\text { ingle- } \\
\text { se }\end{array}$ & $\begin{array}{l}\text { france- } \\
\text { se }\end{array}$ & $\begin{array}{l}\text { porto- } \\
\text { ghese }\end{array}$ & $\begin{array}{l}\text { spag- } \\
\text { nolo }\end{array}$ & tedesco & russo & cinese & arabo & $\begin{array}{l}\text { giappo- } \\
\text { nese }\end{array}$ \\
\hline Torino $^{8}$ & $\bullet$ & • & - & - & - & - & - & - & - \\
\hline Langhe $^{9}$ & $\bullet$ & $\bullet$ & - & - & $\bullet$ & - & - & - & - \\
\hline $\begin{array}{l}\text { Lago di } \\
\text { Garda }^{10}\end{array}$ & $\bullet$ & & - & - & $\bullet$ & - & - & - & - \\
\hline Milano $^{11}$ & $\bullet$ & • & $\bullet$ & $\bullet$ & • & $\bullet$ & $\bullet$ & - & - \\
\hline Venezia $^{12}$ & $\bullet$ & $\bullet$ & - & $\bullet$ & $\bullet$ & $\bullet$ & - & - & - \\
\hline Firenze $^{13}$ & $\bullet$ & - & - & - & - & & & - & - \\
\hline Perugia $^{14}$ & $\bullet$ & - & - & - & - & $\bullet$ & • & - & - \\
\hline Roma $^{15}$ & $\bullet$ & • & - & • & • & $\bullet$ & • & • & $\bullet$ \\
\hline Napoli' ${ }^{16}$ & $\bullet$ & $\bullet$ & - & - & • & $\bullet$ & - & - & $\bullet$ \\
\hline Palermo $^{17}$ & - & - & - & - & - & - & - & - & - \\
\hline
\end{tabular}

Si osserva una certa tendenza, soprattutto per destinazioni meno note come Perugia, o ad alto tasso di turismo internazionale (Roma), a privilegiare strategie di localizzazione rivolte a parlanti che rappresentano flussi turistici consistenti o in costante aumento (russo, cinese, arabo). Non disponendo di dati sugli accessi ai siti non siamo

8 https://www.turismotorino.org [22.07.2018].

9 http://www.langheroero.it/ [22.07.2018].

10 http://www.visitgarda.com/it/lago_di_garda/ [22.07.2018].

11 https://www.turismo.milano.it/wps/portal/tur/it [22.07.2018].

12 http://www.veneziaunica.it/ [22.07.2018].

13 http://www.firenzeturismo.it/it/ [22.07.2018].

$14 \mathrm{http}: / /$ turismo.comune.perugia.it/ [22.07.2018].

15 http://www.turismoroma.it/ [22.07.2018].

$16 \mathrm{http://www.inaples.it/} \mathrm{[22.07.2018].}$

17 http://www.provincia.palermo.it/turismo/, https://turismo.comune.palermo.it/ [22.07.2018]. 
in grado di verificare se i turisti stranieri prediligano, per informarsi sulla meta (prima e durante il viaggio), i portali ufficiali o utilizzino altri canali di informazione, prodotti nella loro lingua e nel paese d'origine (cfr. ad es. i canali social curati dall'ENIT in lingua tedesca, ENIT-Germania 2017). Certamente la presenza di uno spettro diversificato di lingue di comunicazione testimonia da un lato il ruolo della contiguità territoriale e della lingua del vicino per la scelta delle lingue di comunicazione (es. Torino - francese), dall'altro la presenza di scelte linguistiche che rivelano la consapevolezza del ruolo economico del multilinguismo (cfr. Gazzola 2016). Tale attenzione emerge dal documento programmatico del MiBACT sul Piano strategico per la digitalizzazione del turismo (2017), in cui si fanno considerazioni che agli esperti possono sembrare alquanto ovvie, ma che per i non addetti ai lavori così ovvie non sono:

"Particolare attenzione dovrà essere dedicata agli aspetti di internazionalizzazione e localizzazione: ciascun mercato di riferimento ha delle peculiarità socio-linguistiche specifiche ed è fondamentale che venga affrontato con registri comunicativi, strumenti e canali adeguati. In questo senso l'internazionalizzazione non può più essere ridotta ad una semplice opera di traduzione linguistico-grammaticale. È necessario infatti interpretare le aspettative ed i bisogni del turista alla luce delle consuetudini sociali del particolare mercato di riferimento al quale appartiene, e puntare su una comunicazione declinata per quelle specifiche caratteristiche. Per esempio, in ottica SEO, tradurre letteralmente keywords non è considerabile un best practice di internazionalizzazione: l'utilizzo della traduzione letterale del lemma motorino in Cina è molto meno efficace di quello di Vespa". ${ }^{18}$

\section{Il tedesco nella comunicazione turistica in Piemonte}

Per quanto riguarda i flussi turistici in Piemonte, una regione che si è aperta relativamente tardi al turismo internazionale rispetto ad altre regioni italiane, la Germania è il primo mercato estero e rappresenta circa il 23\% delle presenze straniere ${ }^{19}$. Come si può osservare in Tab. 2, le Aziende turistiche locali (ATL) propongono informazioni promozionali in più lingue, anche se le strategie di localizzazione sono molto eterogenee e variano da un minimum (ATL Torino) a una gamma più ampia di lingue di comunicazione, evidentemente scelte in base ai principali bacini di utenza o con l'intenzione di intercettare turisti provenienti da aree emergenti (l'ATL di Alessandria

18 http://www.beniculturali.it/mibac/multimedia/MiBAC/documents/1460024515998_TD_Lab.pdf, p. 73 [22.07.2018].

19 Fonte: Osservatorio turistico regionale, www.visitpiemonte.it [14.04.2018]. 
propone alcuni contenuti in lingua cinese, quella di Novara offre una selezione di pagine in lingua giapponese). La lingua tedesca è lingua di localizzazione per tutte le ATL del territorio piemontese ad eccezione di quella di Torino.

Tab. 2: Scelte linguistiche dei portali informativi delle ATL piemontesi (12/06/2018).

\begin{tabular}{|c|c|c|c|c|c|c|c|}
\hline & inglese & francese & spagnolo & russo & tedesco & cinese & giapponese \\
\hline Alessandria & $\bullet$ & $\bullet$ & - & - & $\bullet$ & $\bullet$ & - \\
\hline Asti & $\bullet$ & $\bullet$ & $\bullet$ & $\bullet$ & $\bullet$ & - & - \\
\hline Cuneo & $\bullet$ & $\bullet$ & $\bullet$ & - & $\bullet$ & - & - \\
\hline Langhe & - & $\bullet$ & - & - & $\bullet$ & - & - \\
\hline Novara & $\bullet$ & $\bullet$ & $\bullet$ & - & $\bullet$ & $\bullet$ & $\bullet$ \\
\hline Torino & $\bullet$ & $\bullet$ & - & - & - & - & - \\
\hline Valsesia-Vercelli & - & - & - & - & - & - & - \\
\hline
\end{tabular}

La consapevolezza della centralità della lingua materna dei potenziali turisti per la promozione del territorio si osserva anche in portali informativi di destinazioni di nicchia del territorio piemontese: ad es. la localizzazione in lingua tedesca del sito promozionale dei comuni della remota Val Maira intercetta l'interesse sempre più consistente dei turisti tedescofoni per questa valle. Anche le scelte linguistiche del portale di ecoturismo Sweet mountains (www.sweetmountains.it), contenente un database su ospitalità e attività di turismo sostenibile nell'arco alpino piemontese e valdostano con traduzioni localizzate in tre lingue (inglese, tedesco, francese), testimoniano l'importanza della formulazione di contenuti in diverse lingue per agevolare e orientare a proprio favore la fase di ricerca e scelta della meta.

Nel progetto "Sprechen Sie Deutsch? Il tedesco come lingua del turismo in Piemonte" ${ }^{20}$ l'attenzione è incentrata sulle attività comunicative scritte e orali nella fase on-trip e dunque sul contatto linguistico fra turisti tedescofoni e locali. Il progetto ha lo scopo di rilevare gli effettivi fabbisogni linguistici sul territorio attraverso interviste e questionari sulle scelte linguistiche nell'interazione con turisti tedescofoni e sulla auto- e eteropercezione delle competenze linguistiche, coinvolgendo sia i soggetti impegnati nell'assistenza e accoglienza dei turisti (agenzie di viaggi, tour operator, ATL territoriali, hotel, esercizi ricettivi di vario tipo ecc.) sia i turisti stessi. La decisione di raccogliere dati da entrambi i 'fronti' è legata alla necessità di colmare un'importante lacuna presente nelle poche ricerche esistenti, che considerano esclusivamente i feedback dei professionisti del settore e non il riscontro da parte di chi rappresenta il vero fulcro dell'esperienza turistica, ovvero il turista (cfr. i dati raccolti nel progetto

20 Progetto coordinato da M. Costa, in collaborazione con il Goethe Institut di Torino. 
SLEST e Blue/Harun 2003 sul concetto di "hospitality language”). Inoltre, incrociando e mettendo a confronto i risultati dell'indagine, le risposte e i punti di vista dei due 'attori' (operatori e visitatori) si potrà ottenere una visione d'insieme sulla percezione delle necessità linguistiche, da una parte, e sulle effettive esigenze, dall'altra, ottenendo così un panorama più reale, completo e attendibile sul fabbisogno linguistico nel territorio preso in considerazione. In una fase successiva il progetto prevede di inventariare e analizzare la produzione di testi scritti/digitati destinati ai turisti tedescofoni e le interazioni fra turisti e locali in lingua tedesca. L'analisi linguisticodiscorsiva dei generi e delle situazioni comunicative ricorrenti servirà per formulare il bi-sogno formativo proprio a partire dall'analisi in situ, individuare le competenze comunicative da sviluppare in fase di formazione e progettare materiali per la formazione di personale qualificato nella relazione con il pubblico in lingua tedesca.

\section{Bibliografia}

Ammon, Ulrich (2015): Die Stellung der deutschen Sprache in der Welt. Berlin/München/Boston: de Gruyter.

Blue, George M./Harun, Minah (2003): Hospitality language as a professional skill. In: English for Specific Purposes 22, 73-91.

Calvi, Maria Vittoria (2004): El lenguaje del turismo en las pàginas web de los Paradores. In: van Hooft Comajuncosas, Andreu (ed.): Textos y Discursos de Especialidad. Amsterdam: Rodopi, 61-70.

Calvi, Maria Vittoria (2006): Lengua y comunicación en el español del turismo. Madrid: Arco/Libros. Costa, Marcella/Ravetto, Miriam (2018): Asymmetries and adaptation in guided tours with German as a foreign language. In: Held, Gudrun (ed.): Strategies of adaptation in tourist communication. Linguistic insights. Amsterdam: Brill, 145-160.

Costa, Marcella/Müller-Jacquier, Bernd (eds.) (2010): Deutschland als fremde Kultur. Vermittlungsverfahren in Touristenführungen. München: Iudicium.

Dann, Graham (1996): The language of tourism: A sociolinguistic perspective. Wallingford: Cab International.

Dannerer, Monika/Franz, Marianne/Ortner, Heike (2017): „Da pendelt sich die Sprache automatisch so ein “: Sprachliche Identität, Akkommodation und Management von innerer und äußerer Mehrsprachigkeit bei Tiroler Privatvermietern. In: Zeitschrift für Angewandte Linguistik 67, 115-147.

Duchêne, Alexandre (2009): Marketing, management and performance: multilingualism as commodity in a tourism call centre. In: Language Policy 8, 27-50.

Duchêne, Alexandre/Piller, Ingrid (2011): Mehrsprachigkeit als Wirtschaftsgut: Sprachliche Ideologien und Praktiken in der Tourismusindustrie. In: Kreis, Georg (ed.): Babylon Europa. Zur europäischen Sprachlandschaft. Basel: Schwabe Verlag, 135-157.

ENIT - Germania (2017): Germania. Rapporto congiunto Ambasciata/Consolati/ENIT. http://www.enit.it/it/studi/focus-paese/category/6-rapporti-enitmae-rapporti-enitmae-europa.html [22.07.2018].

Fandrych, Christian/Thurmair, Maria (2010): Orientierung im Kulturraum: Reiseführertexte und Audio-Guides. In: Costa, Marcella/Müller-Jacquier, Bernd (eds.): Deutschland als fremde Kultur: Vermittlungsverfahren in Touristenführungen. München: Iudicium, 163-188. 
Gazzola Michele (2016): Il valore economico delle lingue. In: Gobbo, Federico (ed.): Lingua, politica, cultura: Serta gratulatoria in honorem Renato Corsetti. New York: Mondial, 47-54.

Goethals, Patrick (2014): Linguistic accommodation in professional tourism contexts. A didactic approach based on tourists' testimonials. In: Iberica 28, 181-202.

Goethals, Patrick (2016): Multilingualism and international tourism: a content- and discourse-based approach to language-related judgments in web 2.0 hotel reviews. In: Language and Intercultural Communication 16, 2, 235-253.

Gotti, Maurizio (2007): The language of tourism as specialized discourse. In: Palusci, Oriana (ed.): Translating tourism. Linguistic and cultural representations. Trient: Editrice Università degli Studi di Trento, 15-34.

Held, Gudrun (ed.) (2018): Strategies of Adaptation in Tourist Communication. Linguistic Insights. Leiden/Boston: Brill.

Heller, Monica (2010): The Commodification of Language. In: Annual Review of Anthropology 39, 1, 101-114.

Jaworski, Adam/Thurlow, Crispin (2010): Language and the globalizing habitus of tourism: Towards a sociolinguistics of fleeting relationships. In: Coupland, Nikolas (ed.): Handbook of language and globalisation. Oxford, U.K.: Wiley-Blackwell, 255-286.

Maci, Stefania (2007): Virtual touring. The web-language of tourism. In: Linguistica e filologia 25, 41-65.

Nigro, Maria Giovanna (2005): The language of tourism as LSP? A corpus-based study of the discourse of guidebooks. In: Picht, Heribert (ed.): Modern Approaches to Terminological Theories and Applications. Frankfurt: Peter Lang, 187-198.

Reuter, Ewald (2011): DaF im Tourismus - Tourismus im DaF-Unterricht. In: German as a foreign language 3, 3-32.

SLEST: http://slest2-0.eu/de [22.07.2018]

Wilson, Adam (2018): The local language of tourism in international tourist information encounters: adapting the what and the how. In: Held, Gudrun (ed.): Strategies of adaptation in tourist communication. Leiden: Brill. doi: https://doi.org/10.1163/9789004359574_008 
Brought to you by | Universita Studi di Torino 